\title{
Biomaterial bridges enable regeneration and re-entry of corticospinal tract axons into the caudal spinal cord after SCI: Association with recovery of forelimb function
}

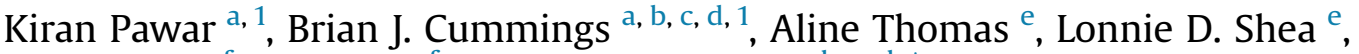 \\ Ariel Levine ${ }^{\mathrm{f}}$, Sam Pfaff ${ }^{\mathrm{f}}$, Aileen J. Anderson ${ }^{\mathrm{a}, \mathrm{b}, \mathrm{c}, \mathrm{d}, *}$ \\ a Institute for Memory Impairments and Neurological Disorders (iMIND), University of California, Irvine, CA, USA \\ b Sue and Bill Gross Stem Cell Research Center, University of California, Irvine, USA \\ ${ }^{c}$ Department of Physical Medicine and Rehabilitation, University of California, Irvine, CA, USA \\ ${ }^{\mathrm{d}}$ Department of Anatomy and Neurobiology, University of California, Irvine, CA, USA \\ e Department of Chemical and Biological Engineering, McCormick School of Engineering, Northwestern University, Evanston, IL, USA \\ ${ }^{\mathrm{f}}$ Salk Institute, San Diego, USA
}

\section{A R T I C L E I N F O}

Article history:

Received 11 May 2015

Accepted 18 May 2015

Available online 23 June 2015

\section{Keywords:}

Spinal cord injury

Bridge

Poly(lactide-co-glycolide) (PLG)

Nerve guide

Regeneration

Macrophage

Growth associated protein 43 (GAP-43)

Ladder beam

Cylinder reaching

\begin{abstract}
A B S T R A C T
Severed axon tracts fail to exhibit robust or spontaneous regeneration after spinal cord injury (SCI). Regeneration failure reflects a combination of factors, including the growth state of neuronal cell bodies and the regeneration-inhibitory environment of the central nervous system. However, while spared circuitry can be retrained, target reinnervation depends on longitudinally directed regeneration of transected axons. This study describes a biodegradable implant using poly(lactide-co-glycolide) (PLG) bridges as a carrier scaffold to support regeneration after injury. In order to detect regeneration of descending neuronal tracts into the bridge, and beyond into intact caudal parenchyma, we developed a mouse cervical implantation model and employed Crym:GFP transgenic mice. Characterization of Crym:GFP mice revealed that descending tracts, including the corticospinal tract, were labeled by green fluorescent protein (GFP), while ascending sensory neurons and fibers were not. Robust co-localization between GFP and neurofilament-200 (NF-200) as well as GFP and GAP-43 was observed at both the rostral and caudal bridge/tissue interface. No evidence of similar regeneration was observed in mice that received gelfoam at the lesion site as controls. Minimal co-localization between GFP reporter labeling and macrophage markers was observed. Taken together, these data suggest that axons originating from descending fiber tracts regenerated, entered into the PLG bridge at the rostral margin, continued through the bridge site, and exited to re-enter host tissue at the caudal edge of the intact bridge. Finally, regeneration through implanted bridges was associated with a reduction in ipsilateral forelimb errors on a horizontal ladder task.
\end{abstract}

Published by Elsevier Ltd.

\section{Introduction}

Many factors contribute to the pathogenesis of SCI, including axonal damage, dysfunction, and degeneration. Axons originating in descending cortical tracts exhibit dieback over one or more spinal segments but do not degenerate back to the cell body after

\footnotetext{
* Corresponding author. University of California, Irvine 2030, Sue \& Bill Gross Stem Cell Research Center, Irvine, CA 92697-1705, USA

E-mail address: aja@uci.edu (A.J. Anderson).

1 These authors contributed equally.
}

spinal cord injury (SCI) [1-3], hence spared axons are readily found rostral to the lesion. However, although there is compelling evidence for spontaneous axonal plasticity and collateral formation in the reorganization of spinal circuitry after SCI [4-7], descending CNS axons do not spontaneously regenerate across the lesion site to yield functional reconnections [8]. Axon regeneration in the CNS is restricted by a combination of intrinsic and extrinsic factors, including the reduced capacity of the neuronal cell body to sustain a growth activated state, formation of a glial scar, and deposition of factors inhibitory to axonal growth at the injury site; for example, chondroitin sulfate proteoglycans and myelin-associated inhibitors of regeneration [9]. Accordingly, while not the only target for repair, 
investigation of methods to enhance or enable the regeneration of spared axons, with the goal of promoting functional recovery, has remained an area of intensive research [9-12].

Early experiments using peripheral nerve grafts (PNG) suggested that both graft architecture and the cells within the graft enable axonal growth $[13,14]$. One strategy to enhance regenerative axonal growth post-SCI has thus been a biomaterials approach, in which a bridge or scaffold material is implanted at the lesion epicenter to provide a substrate for axonal growth and guidance [15]. One biomaterials approach has focused on injectable compounds that can fill the SCI cystic cavity and increase axon growth into or adjacent to the scaffold [16-21]. A second biomaterials approach has sought to direct axonal growth through the damaged region of the spinal cord using $3 \mathrm{D}$ structured bridges that incorporate guidance conduits or channels [22-29]. In this regard, we have previously reported the development of porous, multichannel poly(lactide-co-glycolide) (PLG) bridges in a rat thoracic SCI model [28-31]. In these studies, PLG bridges with linear channels and interconnected pores in vivo showed rapid ingrowth of cells and filled the space normally occupied by a cystic cavity, stabilizing the injury site. Infiltrating cells of multiple types including macrophages and Schwann cells were observed in high numbers, in a manner affected by bridge porosity. Additionally, glial scar formation proximal to the site of PLG bridge implantation was minimized, perhaps contributing to a more permissive environment for axonal regeneration [9]. In parallel with these observations, neurofilament (NF-200), choline acetyl transferase (ChAT), and Calcitonin Gene-Related Peptide (CGRP) positive axons were observed to enter and extend for long distances with a defined orientation within the bridge channels, suggesting the potential of both descending motor and ascending sensory fibers to enter the bridge matrix and exhibit regenerative growth [28,29,31].

Previous studies have suggested that biomaterials can enhance regeneration of descending spinal cord axons through the injury epicenter in association with increased tissue sparing at the lesion site [32]. However, only one study has reported regeneration not only into a biomaterial scaffold, but exiting that scaffold into the intact parenchyma of the spinal cord caudal to the site of implantation [33]. Furthermore, only one study employing either of the biomaterials approaches discussed here has reported functional motor recovery in an SCI model in comparison with no-bridge control conditions in the absence of exogenous cells or trophic factors. That study injected salmon fibrin into a unilateral hemisection model in comparison with human fibrin or no treatment, and reported that recovery was associated with immunohistochemical evidence for serotonergic fiber sprouting adjacent to, but not through the bridge material [20].

While immunohistochemical detection of anti-neurofilament and neurotransmitter antibodies is highly sensitive, in most cases this method does not permit identification of the projection origin for labeled axons and fibers. The latter goal has traditionally relied upon the injection of anterograde or retrograde neuroanatomical tracers, which are dependent upon transport or diffusion of injected tracer and have several limitations in vivo. First, tracer injection is associated with both variability and non-specific diffusion; critically, non-specific diffusion of compounds such as fluorogold is enhanced in implanted biomaterial scaffolds. Second, labeling by tracer injection is unavoidably incomplete; for example, only $25 \%$ of corticospinal tract (CST) axons are estimated to transport small molecule tracers such as BDA, which can mean that small fibers that may nonetheless be functionally important fail to be evaluated [34-36]. To overcome these and other limitations in live preparations, transgenic mice expressing fluorescent reporter labels transported in axons have recently been tested as a means to label one or more CNS tracts, e.g. the CST [37,38].
In this study, we sought to specifically investigate the capacity of PLG bridges to create a permissive environment for axon regeneration that could support growth not only into the biomaterial channels, as shown previously, but enable re-entry of regenerated descending fibers into intact spinal cord parenchyma caudal to the implant. We sought to optimize axonal growth potential, sensitivity of detection of functional locomotor improvement, and capacity to utilize genetic tools by establishing a cervical bridge implantation model in the mouse, and testing regeneration in transgenic mice expressing Crym:GFP in descending fiber tracts in the spinal cord. Mu-Crystallin (Crym) is expressed in a diverse array of tissues, including brain, where it is heavily localized within the descending projection neurons of the motor cortex [39-41]. After establishing evidence of bridge apposition and axonal regeneration in our mouse cervical SCI implantation model, and characterizing the specificity of Crym:GFP reporter expression in the spinal cord, we investigated the regeneration of descending axons into and beyond implanted PLG multichannel bridges.

\section{Materials and methods}

\subsection{Multiple channel bridges}

Bridges were fabricated by adapting a previously established gas foaming/particulate leaching procedure [29,31] to include caramelized sucrose fibers as a channel template [28]. The final bridge dimensions were $1.25 \mathrm{~mm}$ in height, $1.15 \mathrm{~mm}$ in length $(\mathrm{R}-\mathrm{C})$, and $0.75 \mathrm{~mm}$ in width $(\mathrm{M}-\mathrm{L})$. The channels were characterized and bridge diameters measured $(n=6)$ using light microscopy images (Leica Microsystems, Wetzlar, Germany) and Image J software. The cross sectional area occupied by the channels and the porosities of these bridges were calculated using equations established previously $[42,43]$.

\subsection{Spinal cord injury}

All animals housing conditions, surgical procedures, and postoperative care was conducted according to IACUC guidelines at the University of California, Irvine. Female mice were anesthetized using isoflurane (2\%). Mice received a C4-C5 laminectomy, followed by a left-sided double lateral hemisection and removal of a unilateral 1-1.1 mm segment of the spinal cord to enable bridge implantation into the resulting gap. A length standard was used to create the hemisection gap, in order to ensure that the length of the bridge would be greater than the length of the hemisection and ensure good apposition of the multichannel bridge to the spared rostral and caudal spinal cord. If the hemisection exceeded $1.1 \mathrm{~mm}$ in length, such that there was not tight apposition to the $1.15 \mathrm{~mm}$ long bridge, that animal was excluded at the time of surgery. PLG multichannel bridges were implanted into the gap immediately after SCI in Crym:GFP C57Bl6 mice (Pfaff laboratory; $\mathrm{N}=5$ ); control C57Bl6 mice (JAX; $\mathrm{N}=5$ ) received gelfoam implanted into the gap immediately after SCI. No animals were excluded from either behavioral or histological assessments. Following randomized implantation of either a bridge or gelfoam within the gap, the dorsal surface of the spinal cord above the injury site was covered with gelfoam, the muscle sutured with 5-0 chromic gut, and the skin closed using wound clips. Postoperative care included administration of Baytril (enrofloxacin $2.5 \mathrm{mg} / \mathrm{kg}$, once a day for 2 weeks), buprenorphine $(0.1 \mathrm{mg} / \mathrm{kg}$ twice a day for 3 days $)$, and lactate ringer solution ( $5 \mathrm{ml} / 100 \mathrm{~g}$, once a day for 5 days). Bladders were expressed twice daily until function recovered, and mice monitored daily thereafter. 


\subsection{Behavioral tests}

A total of 2-3 weeks of pre-handling and pre-testing was performed to acclimate the mice to handling and ensure that they exhibited normal locomotor behavior prior to surgery. Mice were tested 1 week before surgery, and at $2 \mathrm{w}, 4 \mathrm{w}$, and $10 \mathrm{w}$ post surgery. For cylinder paw placement, mice were tested in a transparent glass cylinder/beaker for $5 \mathrm{~min}$, with two observers located on opposite sides of the apparatus to enable observation of all movements. Three behaviors were scored: (1) use of the left forelimb alone with weight support; (2) use of the right forelimb alone with weight support; and (3) use of both forelimbs simultaneously or in rapid succession (one paw followed by the other within $0.5 \mathrm{~s}$ ) with weight support. If simultaneous versus independent limb use could not be distinguished, the movement was not scored. For horizontal ladder beam, training consisted of a 5 min acclimation period followed by at least three trials where the animals were directed to run across the ladder beam towards the escape box. Each animal was filmed for a minimum of four trials and scored for errors as described previously [44]. Behavioral data collection and analysis was performed by observers blinded to treatment condition.

\subsection{Perfusion and tissue collection/analysis}

Mice were sacrificed 10 weeks post-lesion/bridge implantation; mice were anesthetized with sodium pentobarbital $(100 \mathrm{mg} / \mathrm{kg}$, i.p.) and transcardially perfused with $30 \mathrm{ml}$ PBS followed by $100 \mathrm{ml}$ 4\% paraformaldehyde. Brains and spinal cords (C3-C7) were dissected and collected. Tissues were post-fixed overnight in $4 \%$ paraformaldehyde, flash frozen at $-65{ }^{\circ} \mathrm{C}$ in isopentane (2-methyl butane), and stored at $-80{ }^{\circ} \mathrm{C}$. Tissues were embedded in optimal cutting temperature compound (OCT, Tissue-Tek), sectioned horizontally at $30 \mu \mathrm{m}$ using a CryoJane tape transfer system and Microm cryostat, and processed for immunohistochemistry as previously described (47). All sections used for immunostaining were from maximal lesion region for consistency in comparison between animals and groups. Briefly, sections were washed in $0.1 \mathrm{M}$ Tris followed by $0.1 \%$ TritonX-100, and blocking with bovine serum albumin (BSA) and normal serum from the species in which the secondary antibody was raised. Sections were incubated overnight in primary antibody (Table 1), followed by incubation with affinitypurified mouse cross-adsorbed donkey anti-rabbit IgG Alexa-Fluor 647 (Millipore) or goat anti-rabbit IgG Alexa-Fluor 647 (Millipore). In the case of F4/80 a direct Alexa Fluor 647 conjugate was used to minimize cross-reactivity. Stained sections were counterstained with Hoechst 33342 (1:1000 Invitrogen). Slides were dried and coversliped using fluoromount G (SouthernBiotech). Negative controls for all immunohistochemical staining were included, and the absence of fluorescent staining following omission of primary antibodies was confirmed for all antibodies and antibody combinations. Images of multi-area $\mathrm{Z}$ stacks were obtained with an Olympus Fluoview FV10i confocal laser scanning microscope using $\times 10, \times 20$, or $\times 60$ water objectives. Maximum intensity projection and stitched images were created using MATL viewer, and processed in Adobe Photoshop. For all markers, all images were captured in a single imaging session using identical settings for

Table 1

List of primary antibodies, sources, and dilutions.

\begin{tabular}{lllll}
\hline Primary antibody & Host & Dilution & Manufacturer & Specificity \\
\hline NF200 & Rabbit & $1: 500$ & Chemicon & Axons and processes \\
F4/80 & Rat & $1: 500$ & SeroTec & Macrophages \\
GAP43 & Rabbit & $1: 500$ & Millipore & Regenerated axons \\
P75 & Rabbit & $1: 1000$ & Abcam & Satellite cells \\
\hline
\end{tabular}

light intensity and camera parameters. For quantification of NF-200 staining, the section (one section/animal) containing the maximum lesion region was identified and used for quantification. NF200 was measured in a defined rectangle $(200 \mu \mathrm{m} \times 200 \mu \mathrm{m})$, and the number of pixels occupied by NF200 within this area determined using Scion Imaging Software (Scion Corp., USA).

\subsection{Statistics}

Results are expressed as mean \pm SEM unless indicated otherwise. For paired comparisons, data were analyzed using Student's t-test. Statistical evaluation was performed using Graphpad Prism 5 for windows (GraphPad Software). Significance levels are indicated as follows: ${ }^{*} \mathrm{p}<0.05 ;{ }^{* *} \mathrm{p}<0.01$.

\section{Results}

\subsection{Bridge implantation and axonal labeling in a mouse cervical SCI implantation model}

Bridges were fabricated using caramelized sucrose fibers as a template for the channels (26); a total of 7 fibers could be packed within the mold, creating a porous, multiple channel bridge (Fig. $1 \mathrm{~A}$ and B). We first tested the feasibility of achieving stable apposition of PLG multichannel bridges with the spared spinal cord parenchyma following a unilateral $\mathrm{C4}-5 \mathrm{SCI}$ in which a 1-1.1 mm segment of the mouse spinal cord (Fig. 1B, D-F) was removed, as described under Methods. As previously observed after thoracic bridge implantation, there was good apposition between the PLG bridge and cervical spinal cord at the time of sacrifice, 10 weeks post-SCI and bridge implantation (Fig. 1C and F).

As expected based on previous results, immunolabeling for NF200 revealed few or no neurofilament positive axons within the region of implantation in gelfoam controls (Fig. 2B), while there was robust detection of NF-200 positive axons within PLG bridges (Figs. 1C and 2E). As described under Methods, for multiple labeling in addition to visualization of the Crym:GFP reporter, pre-adsorbed Fab' fragment fluorescent conjugated secondary antibodies were used in order to minimize background from endogenous IgGs or nonspecific binding to bridge materials, and antibody omission experiments were included to validate the specificity of fluorescent signal and staining. An example of negative staining in the case of omission of the primary antibody is illustrated (Supplemental Fig. $3 \mathrm{~A}$ and $\mathrm{B}$ ). Inclusion of primary antibody showed robust detection of NF-200 positive axons detected using secondary antibody within tissue (Supplemental Fig. 3C).

Consistent with the presence of descending and ascending axons proximal to the site of SCI, NF-200 was observed both above and below the region of implantation in animals receiving either multichannel bridges or gelfoam as a control (Fig. 2A-F). Based on this observation, NF-200 positive axons were quantified at the rostral and caudal borders of animals receiving multichannel bridge versus gelfoam implants. A significant increase in NF-200 labeling was found in bridge-implanted animals in both regions (Fig. 2G and $\mathrm{H}$ ), suggesting axonal preservation adjacent to the site of bridge implantation and/or regeneration both into and out of the bridge in this cervical implantation model.

\subsection{Crym:GFP in the spinal cord identifies descending projections and not ascending DRG sensory axons}

We next focused on characterization of GFP fluorescence in Crym reporter mice with and without PLG bridges. Characterization of Crym:GFP expression in the brain and spinal cord confirmed high levels of GFP in neurons of layer V/VI of the sensorimotor cortex, as 

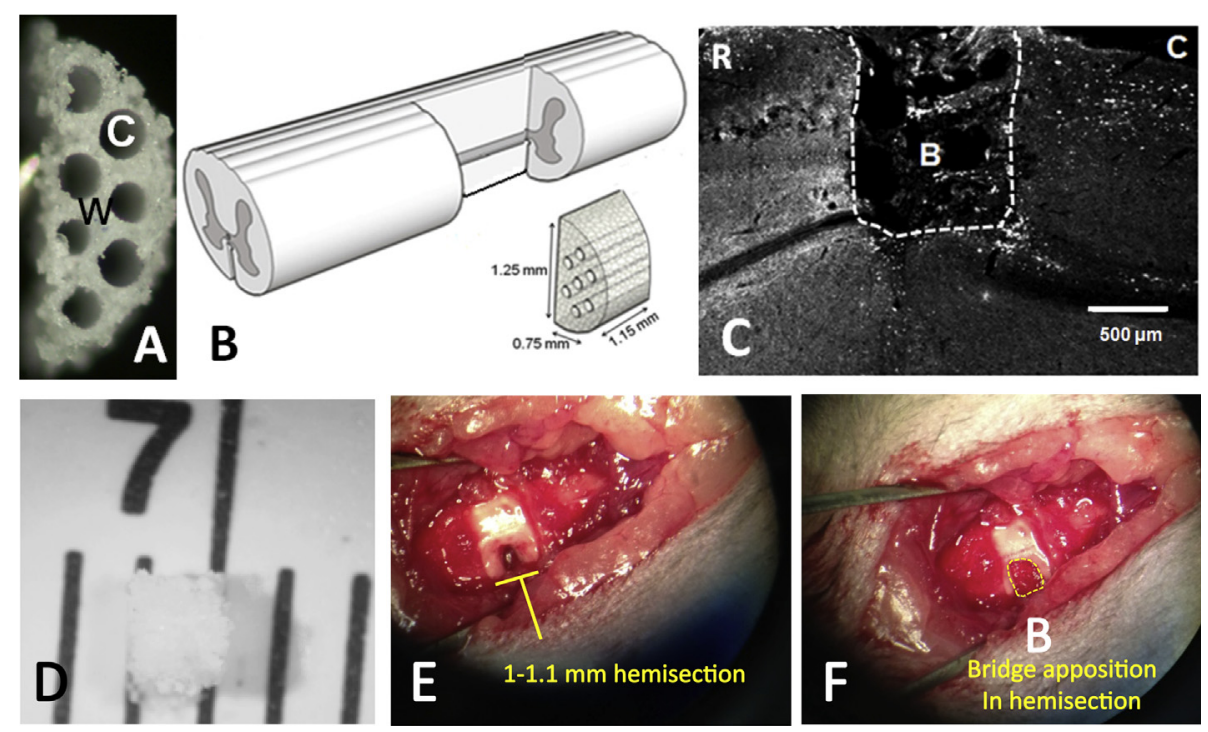

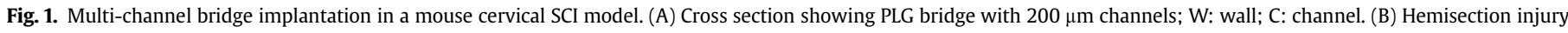

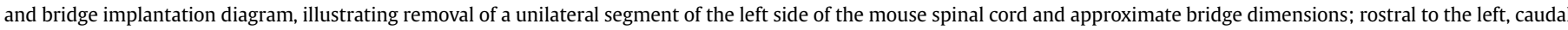

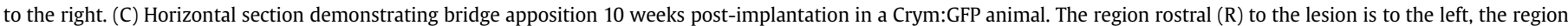

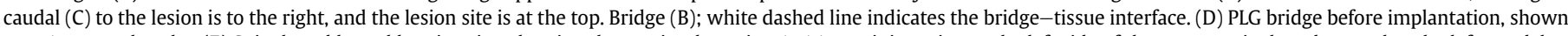

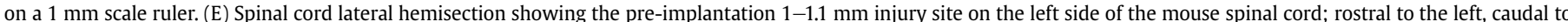

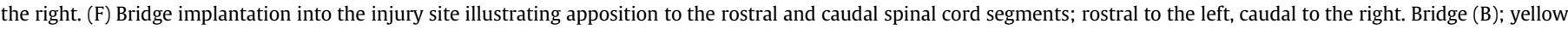
dashed line indicates bridge and lesion area. (For interpretation of the references to colour in this figure legend, the reader is referred to the web version of this article.)
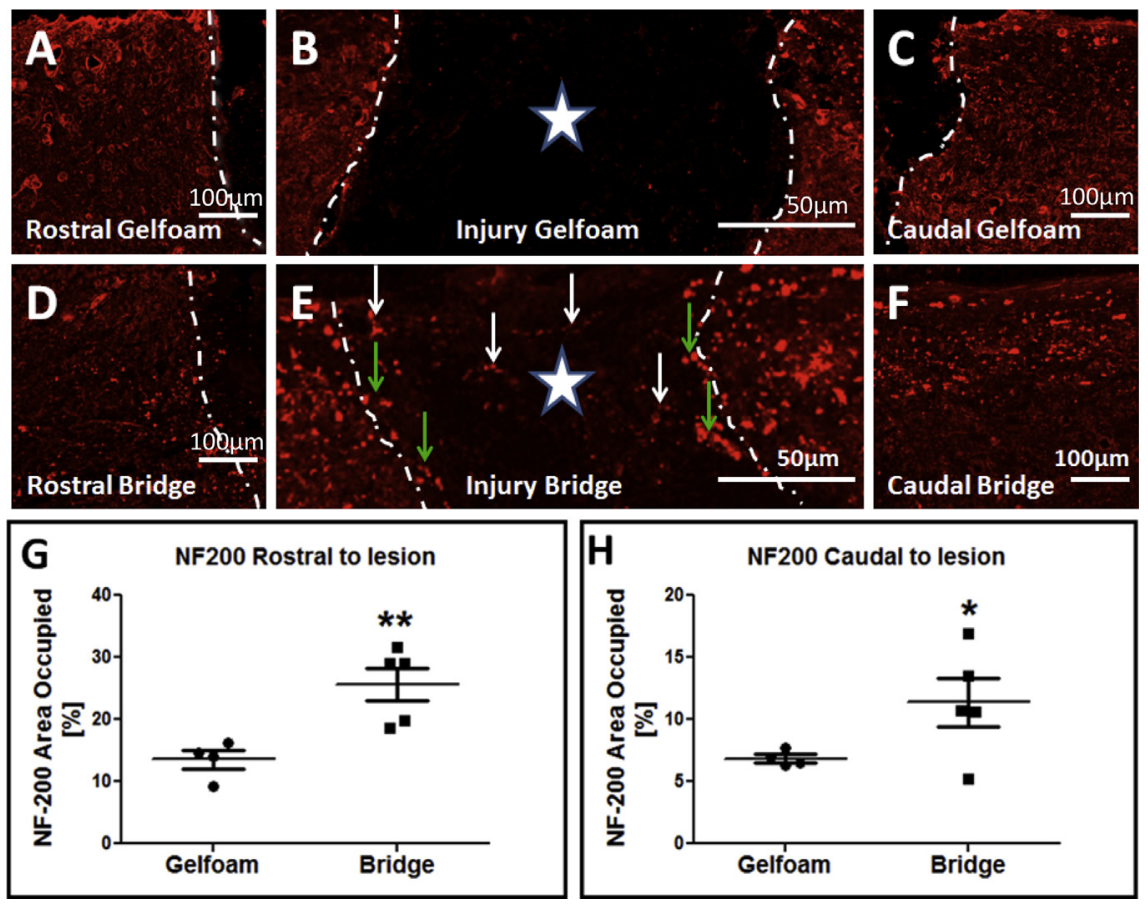

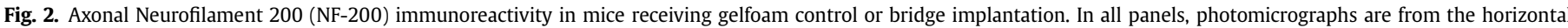

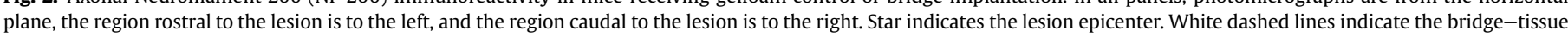

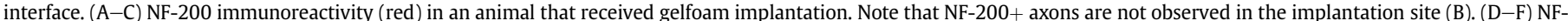

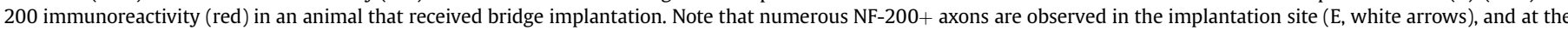

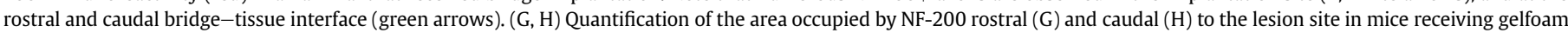

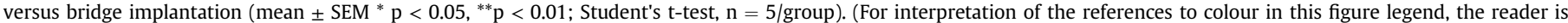
referred to the web version of this article.)

well as in other subcortical regions, including hippocampus (Supplemental Fig. 1A-C). In the spinal cord, Crym:GFP mice exhibited robust labeling of the dorsal column corticospinal tract (CST); GFP+ fibers did not overlap with CGRP+ ascending sensory fibers (red;
Supplemental Fig. 1D). To further confirm that Crym:GFP labeling in the spinal cord did not originate in ascending sensory fibers, we investigated the expression pattern of GFP in relation to p75 and NF-200 labeling in the dorsal root ganglion (DRG). Although p75 
immunolabeled DRG satellite glial cells exhibited co-localization with Crym:GFP (Supplemental Fig. 2A and B), NF-200 or pseudorabies virus positive DRG neurons were GFP-negative (Supplemental Fig. 2C-E), and no Crym:GFP positive ascending sensory axons were observed within or exiting the DRG. These findings demonstrate that Crym:GFP labeling in the spinal cord identifies descending projections, predominantly the dorsal column CST, and not ascending DRG sensory axons.

\subsection{Crym:GFP in and beyond implanted PLG bridges}

Given the localization of Crym:GFP in descending CST axons, we investigated GFP fibers in and beyond implanted PLG bridges. In the mouse, $\sim 90 \%$ of the CST descends in the ventral part of the dorsal columns, with small projections in the dorsolateral and ventral white matter; fibers branch off from this main tract to enter the grey matter of the spinal cord in a segmental fashion. Fig. 3 illustrates the Crym:GFP reporter labeled CST, most strongly visible within the dorsal columns rostral to the bridge, observed as bilateral GFP fluorescence at the midline in horizontal section (Fig. 3A-C large solid white arrows; rostral is to the left in all panels). A, B and C each represent individual animals. In all cases, there was complete or near complete bilateral disruption of the dorsal column CST caudal to the bridge (Fig. 3A-C large open white arrows; caudal is to the right in all panels). Grey and white matter regions are clearly visible in these images; note that numerous Crym:GFP fibers within and caudal to the bridge were located at the lateral edge of the horizontal section, and were not adjacent to potential regions of caudal dorsal column sparing (small white arrows). In contrast, there was no evidence of an increase in GFP+ labeling in the parallel region on the contralateral side of the spinal cord where there was no bridge. At higher power, Cyrm:GFP positive CST fibers were observed to enter the bridge at the rostral interface (Fig. 4A, B, and F), to be localized inside the bridge matrix (Fig. 4A, C, and $G$ ), and to exit the bridge into host tissue at the caudal interface (Fig. 4A, D, E, and H). These findings are consistent with proposed criteria for the demonstration of CNS regeneration [45]. Accordingly, these data suggest that Crym:GFP fibers successfully crossed the bridge and re-entered host tissue, traveling up to $2500 \mu \mathrm{m}$ through the caudal parenchyma (Fig. $4 \mathrm{I}$ ) by 10 weeks post-SCI.

\subsection{Crym:GFP caudal to the bridge does not reflect phagocytosis}

SCI results in a robust inflammatory reaction that includes clearance of debris by infiltrating macrophages/microglia. In order to address whether Crym:GFP labeling observed caudal to the bridge could reflect phagocytosis of degenerating fibers/debris, we conducted immunohistochemistry for $\mathrm{F} 4 / 80$ positive macrophages. F4/80 immunoreactivity (red) was characterized by a rounded and regular morphology, however, only a small fraction of Crym:GFP was co-localized with F4/80 (Fig. 5A-C), suggesting that the majority of Crym:GFP represents intact descending fibers caudal to the site of bridge implantation. This interpretation was further supported by the lack of co-localization between GFP-positive profiles and nuclear Hoechst staining (Fig. 5D-F).

\subsection{Crym:GFP is co-localized with neurofilament}

In order to further test the specificity of Crym:GFP within and caudal to the bridge, we next investigated whether GFP was specifically associated with immunoreactivity for axonal markers using NF-200. NF-200 positive fibers (red) were found in both the rostral (Fig. 6A) and caudal (Fig. 6G) margins of the bridge implantation site in Crym:GFP mice. Crym:GFP+/NF-200+ co-localized fibers (yellow) were observed at the rostral bridge-tissue interface
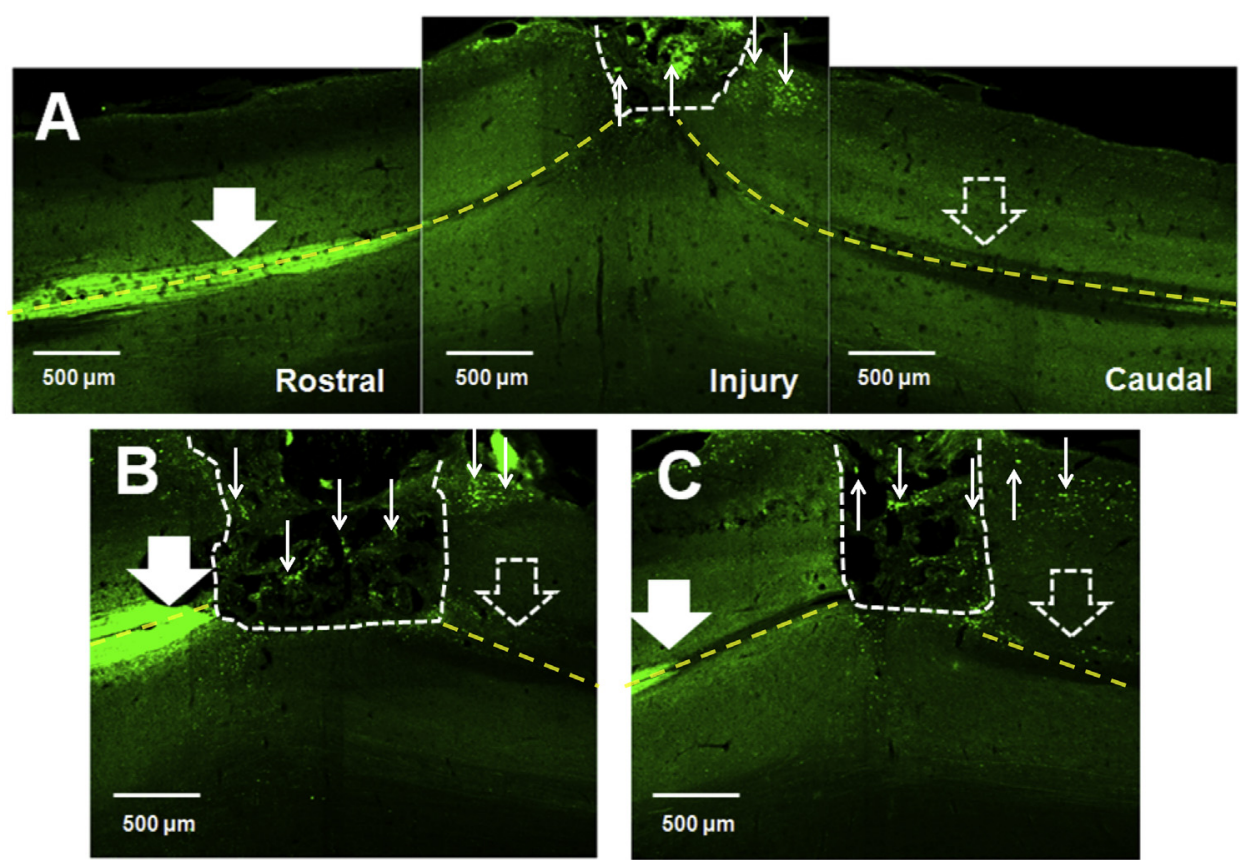

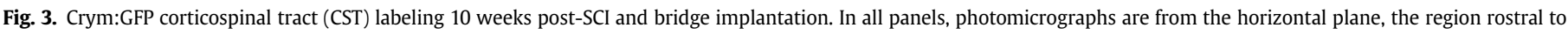

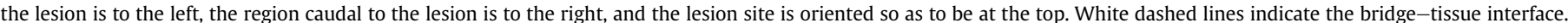

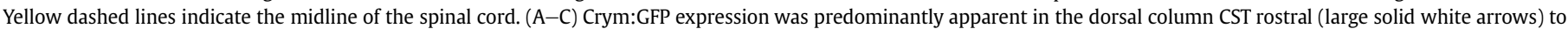

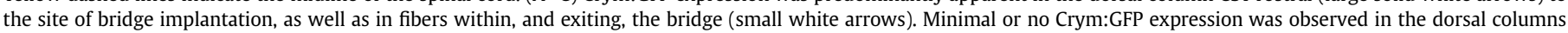

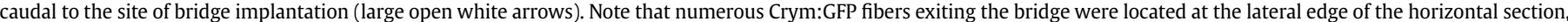

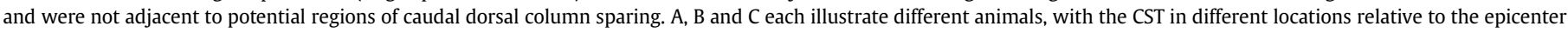
depending on section selection. (For interpretation of the references to colour in this figure legend, the reader is referred to the web version of this article.) 

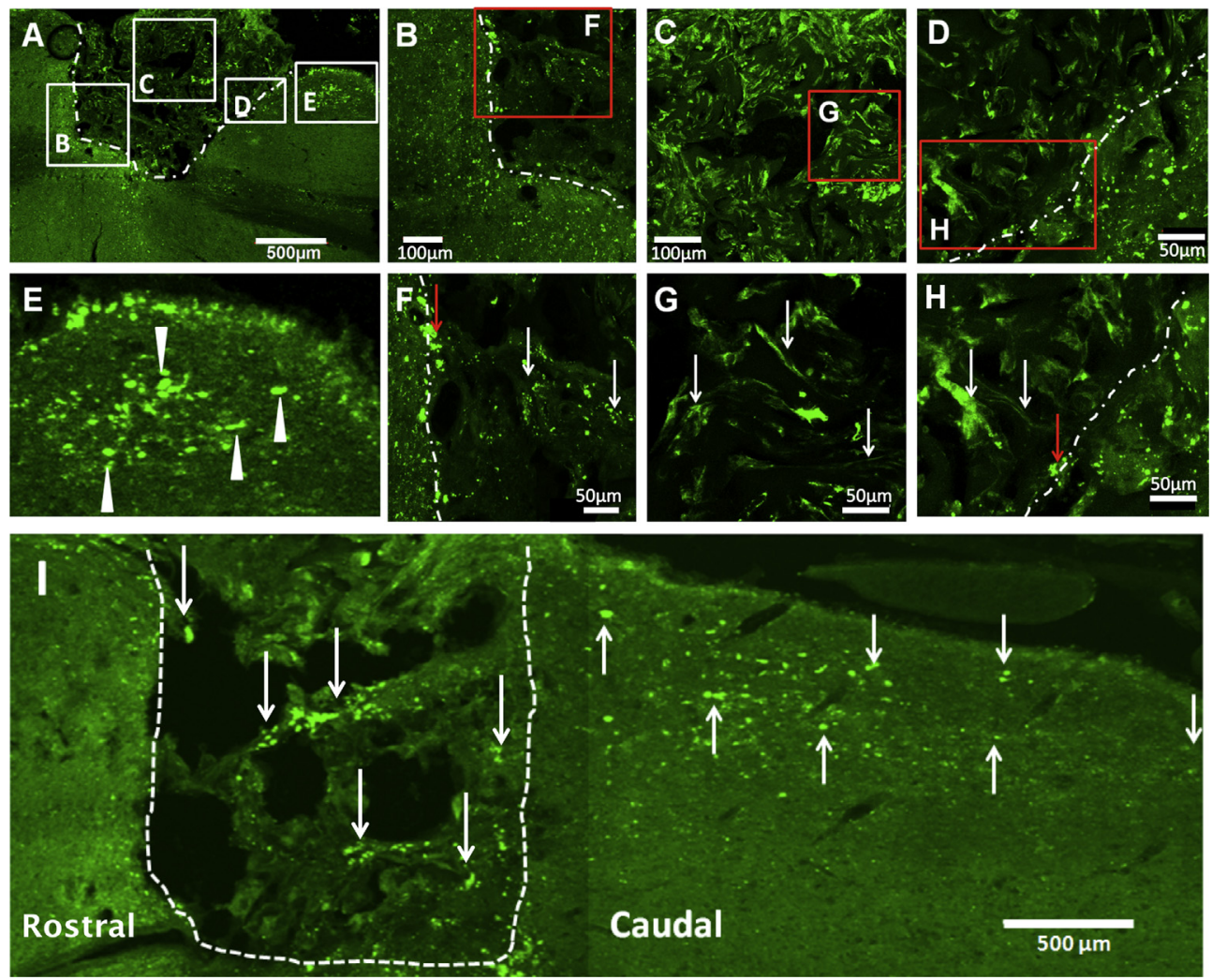

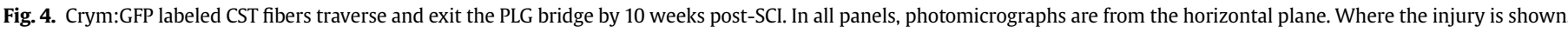

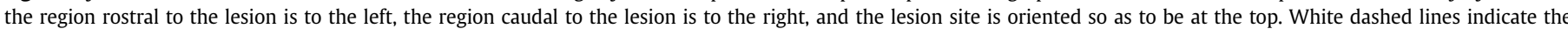

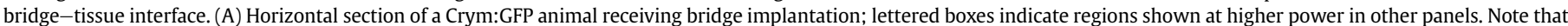

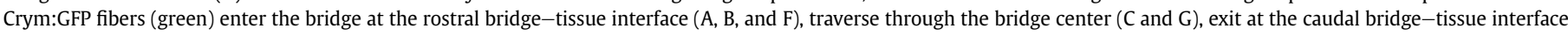

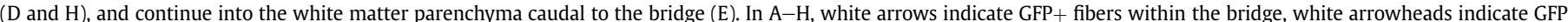

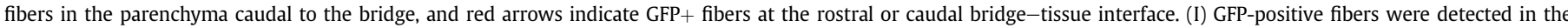

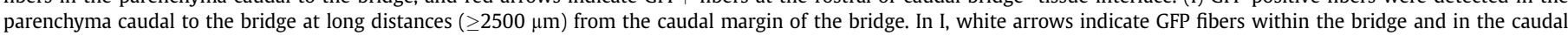
parenchyma. (For interpretation of the references to colour in this figure legend, the reader is referred to the web version of this article.)

(Fig. 6A and B), entering the bridge (Fig. 6B and C), and within the bridge structure (Fig. 6D and E).

Of note, in some cases, Crym:GFP CST fibers entering the bridge appeared to be of contralateral origin. After SCI, it is well documented that there is extensive sprouting of CST projections across the grey matter midline, and that this can mediate spontaneous recovery in hemisection and dorsal column lesion models [46]. In our study, in cases where the hemisection extended across the midline, the implanted bridge still filled this region, as is visible in the horizontal section shown in Fig. 6A (midline indicated by yellow dashed line). In this section, the white and grey matter are clearly distinguishable, and GFP+ fibers (green), as well as Crym:GFP+/NF-200+ co-localized fibers (yellow), from the grey matter contralateral to the bridge/lesion can be observed to approach the border of the bridge and enter it (Fig. 6A and F).

Finally, a significant fraction of Crym:GFP labeling at the caudal bridge-tissue interface was co-localized with NF-200 (Fig. 6G-I), and many Crym:GFP fibers appeared to exit the bridge and re-enter the adjacent spared parenchyma (Fig. $6 \mathrm{~J}-\mathrm{L}$ ). Note that the presence of NF-200+ fibers that are not GFP+ is to be expected in the parenchyma both rostral and caudal to the bridge, as other descending/ascending tracts will be present. Further, the opposite is also true, as other neurofilament subunits may be associated with different growth phases of regenerating fibers. Taken together, these data further support the regeneration of descending axons through the PLG bridge and into the caudal cord.

\subsection{Crym:GFP is co-localized with GAP-43}

To specifically address whether Crym:GFP in and caudal to the bridge reflected regenerating descending fibers, we evaluated GFP co-localization with a specific marker for axonal regeneration, GAP43 (Fig. 7). A significant proportion of Crym:GFP+ fibers (green) within the bridge expressed GAP-43 (red) (Fig. 7A and B), including at the caudal bridge-tissue interface (Fig. 7C and D). Caudal to the bridge, a similar pattern was observed, with robust co-localization of GAP-43+ fibers with Crym:GFP (Fig. 7E-H). Note, however, that GAP-43+ fibers independent of Crym:GFP labeling were also present in the parenchyma caudal to the bridge, consistent with our previous demonstration of the growth of ascending CGRP sensory fibers in this region and within the bridge [47]. Again, these data suggest the regeneration of Crym:GFP+ CST descending axons through the PLG bridge and into the spinal cord caudal to the site of lesion and bridge implantation.

\subsection{Forelimb locomotor function is improved in mice receiving PLG bridges}

Taken together, these data suggest that PLG bridges in a cervical mouse spinal cord lesion model can support the regeneration of descending axons through the PLG bridge and into the caudal parenchyma, which can be detected in Crym:GFP reporter mice. As discussed, we developed this model in order to take advantage of a genetic neuroanatomical tracing strategy, increased regenerative 

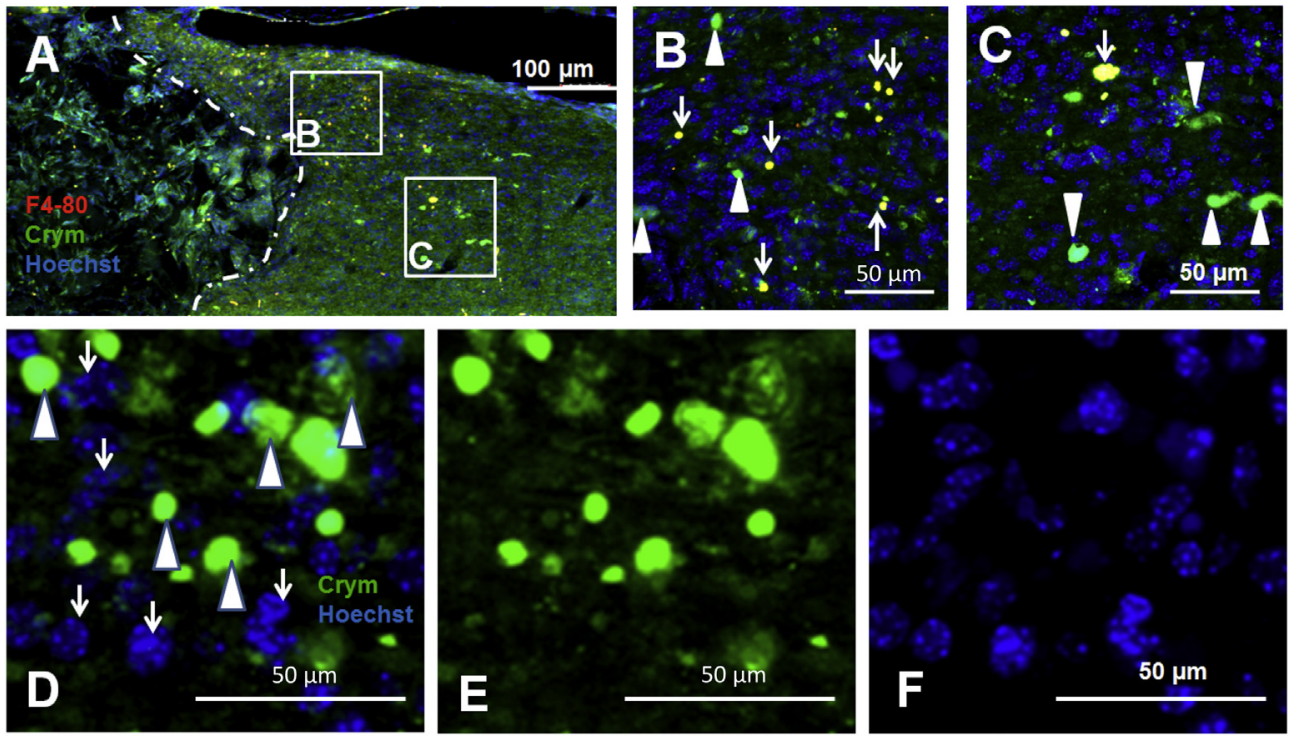

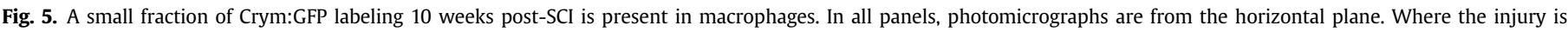

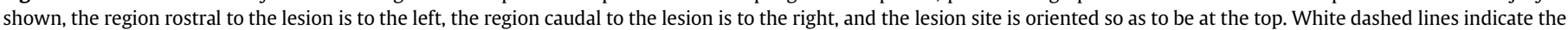

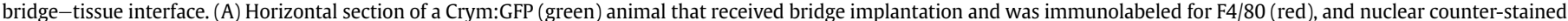

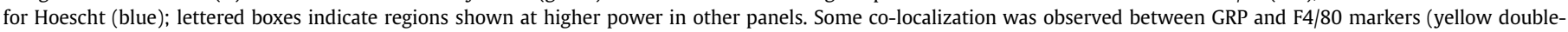

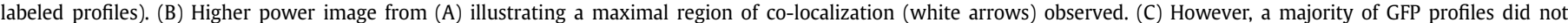

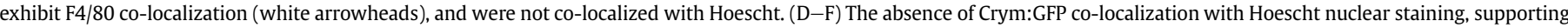

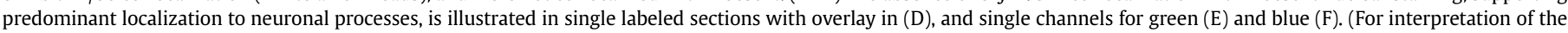
references to colour in this figure legend, the reader is referred to the web version of this article.)

potential in the cervical spinal cord, and more sensitive locomotor assessments in cervical SCI animals. Accordingly, we next tested whether regeneration through a PLG bridge supported forelimb locomotor recovery.

Animals were assessed for motor behavior with a cylinder reaching task and horizontal ladder beam task as described under Methods; all data capture and analyses were conducted by investigators blinded to experimental groups. No evidence of recovery of function was observed in cylinder testing in either the bridge or gelfoam control groups in comparison with pre-injury assessments (Fig. 8A), which is consistent with the disruption of the C5 motor neuron pool in this model. However, while animals receiving bridge implantation performed worse than animals receiving gelfoam at 2 weeks post-implantation (all bridge animals made $100 \%$ errors at 2 -wpi), by 8 weeks post-implantation there was a trend for improvement, and at 10 weeks post-implantation, animals receiving bridge implantation exhibited a significant reduction in ipsilateral forelimb errors on this task versus animals receiving gelfoam (Fig. 8B; one-way ANOVA, F(7,29) p < 0.0001, one-tail t-test $\mathrm{p}=0.02$ ). Critically, spared axons that are displaced or stretched by injury, rather than transected, may transiently lose the capacity to transmit action potentials. This is thought to result in an early loss of function followed by rapid behavioral recovery within days, related to the restoration of neurotransmission in spared fibers. In contrast, recovery associated with regeneration, formation of bridge circuitry, or reconnection with appropriate targets is generally hypothesized to require a delay of several weeks. Accordingly, the timecourse of recovery of function in these experiments is consistent with axonal regeneration as opposed to sparing. These data demonstrate the first evidence for functional recovery following implantation of a $3 \mathrm{D}$ biomaterial scaffold bridge without combination with trophic factor or cell delivery, and suggest that regeneration of descending motor tracts into the spared parenchyma caudal to the SCI lesion site may play a role in this recovery.

\section{Discussion}

More than one million people in the US are currently living with spinal cord injury (SCI) [48]. Nationally, the incidence of cervical SCI exceeds thoracic (51\% vs. 36\%), and the incidence of contusion/ compression trauma exceeds penetrating injuries due to violence (e.g. gun shot or stab wounds). In recent years, translational research for $\mathrm{SCI}$ has been heavily focused on a drive towards restoring function after contusion/compression injuries. In this type of SCI, neuroprotective strategies may be able to limit the initial expansion of injury, and the presence of a rim of spared tissue in $98 \%$ of individuals [49] may permit enhancement of spontaneous recovery by promotion of local sprouting. While penetrating SCI accounts for $14 \%$ of cases annually in the general population [50], it accounts for $28 \%$ of cases in the military population [51], largely due to the contribution of blast injuries. Vertebral burst fractures also often result in physical penetration and damage to spinal cord parenchyma [52]. Critically, injuries that directly sever descending motor and ascending sensory tracts may necessitate a different approach to restoring function than contusion/compression injuries. Moreover, while spontaneous recovery within the first days and months post-SCI is a significant component of final outcome in individuals with contusion/compression injuries [53], the initial deficits resulting from penetrating SCI exhibit substantially less improvement, if any, over time [54]. Little progress has been made in therapeutic strategies for penetrating $\mathrm{SCI}$, in which there may be little opportunity to ameliorate the initial damage, and reconnection of spinal pathways may require a bridge to support true axonal regeneration, as opposed to local sprouting.

As recently reviewed [8], a variety of biomaterial scaffolds have been tested as a means to support central nervous system (CNS) regeneration following injury. However, there has been minimal evidence demonstrating regeneration into and through a scaffold to reach the caudal spared host tissue. The aim of the present study 

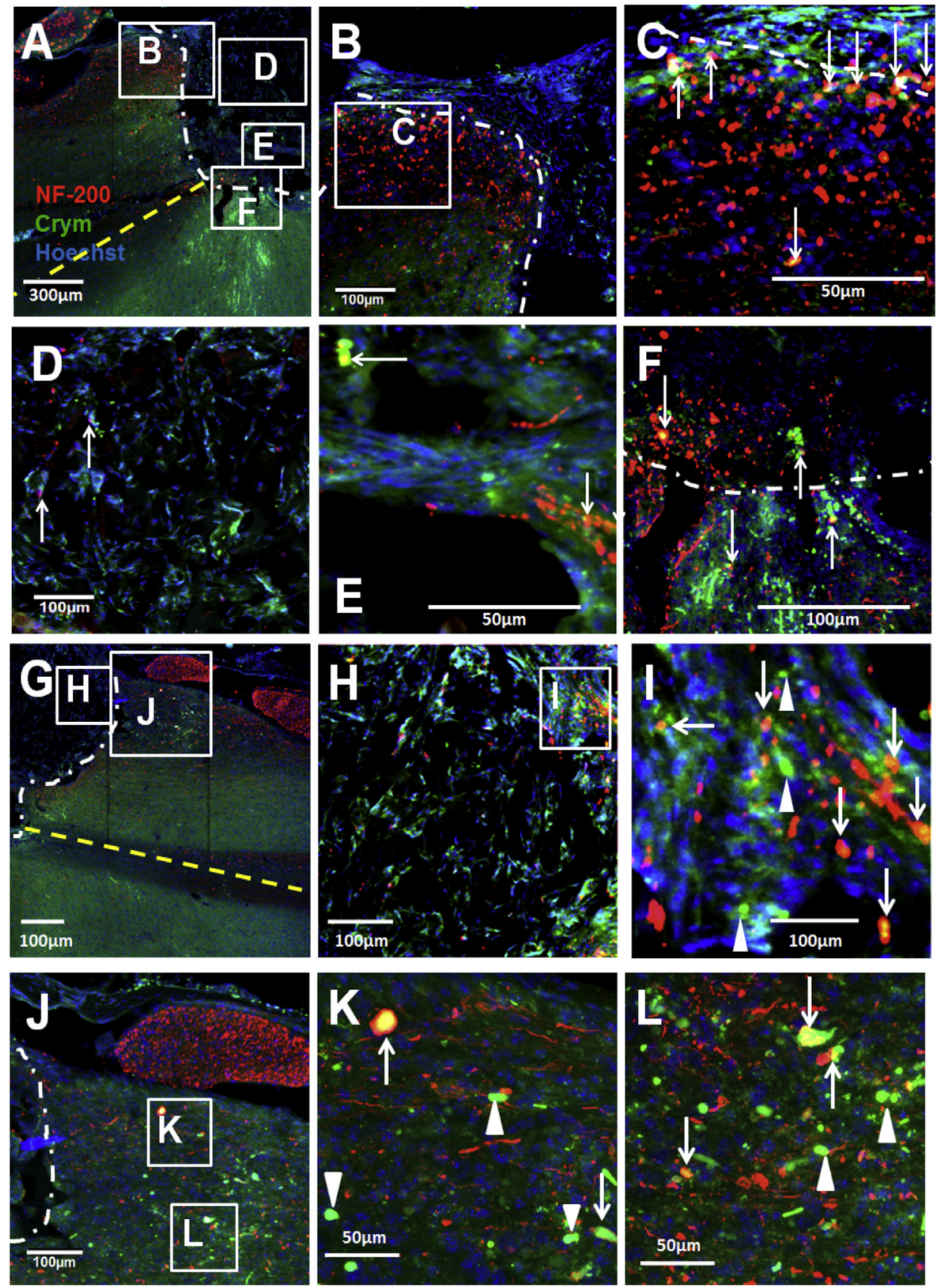

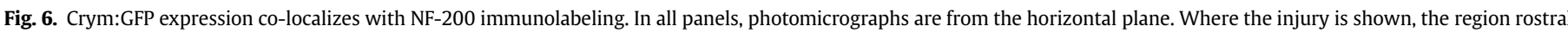

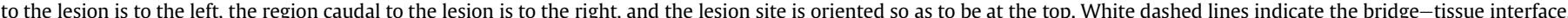

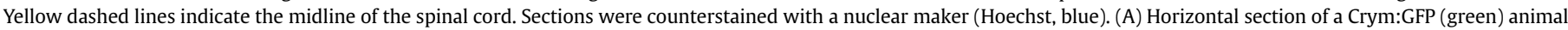

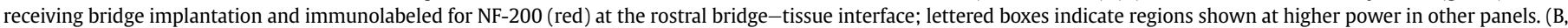

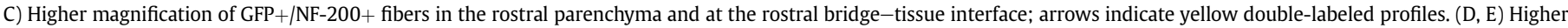

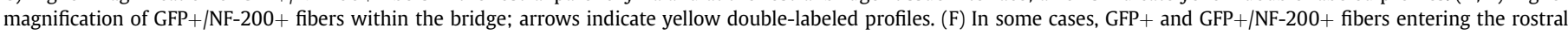

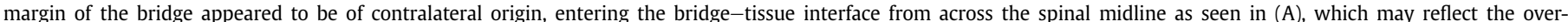

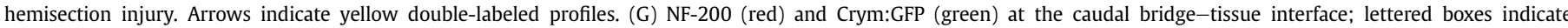

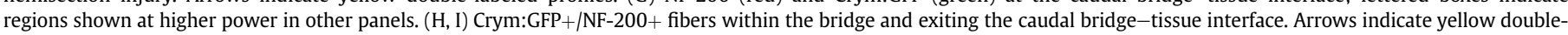

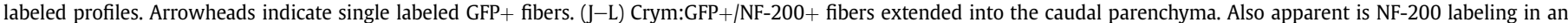

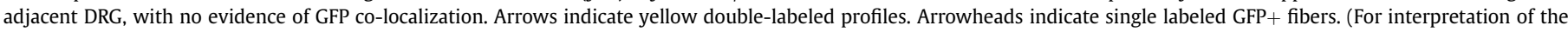
references to colour in this figure legend, the reader is referred to the web version of this article.)

was to evaluate the regeneration of descending axons into and through an implanted PLG bridge with longitudinally oriented channels.

We employed a Crym:GFP transgenic mouse model to reveal descending fiber tracts within the spinal cord. Several similar genetic reporter mice have been used as tools for the assessment of regeneration of descending fiber tracts in the spinal cord [37,55]. In this study, Crym:GFP mice exhibited similar robust labeling of the CST within the spinal cord, with no contribution of ascending sensory fibers to Crym:GFP reporter labeling. Based on the robust 

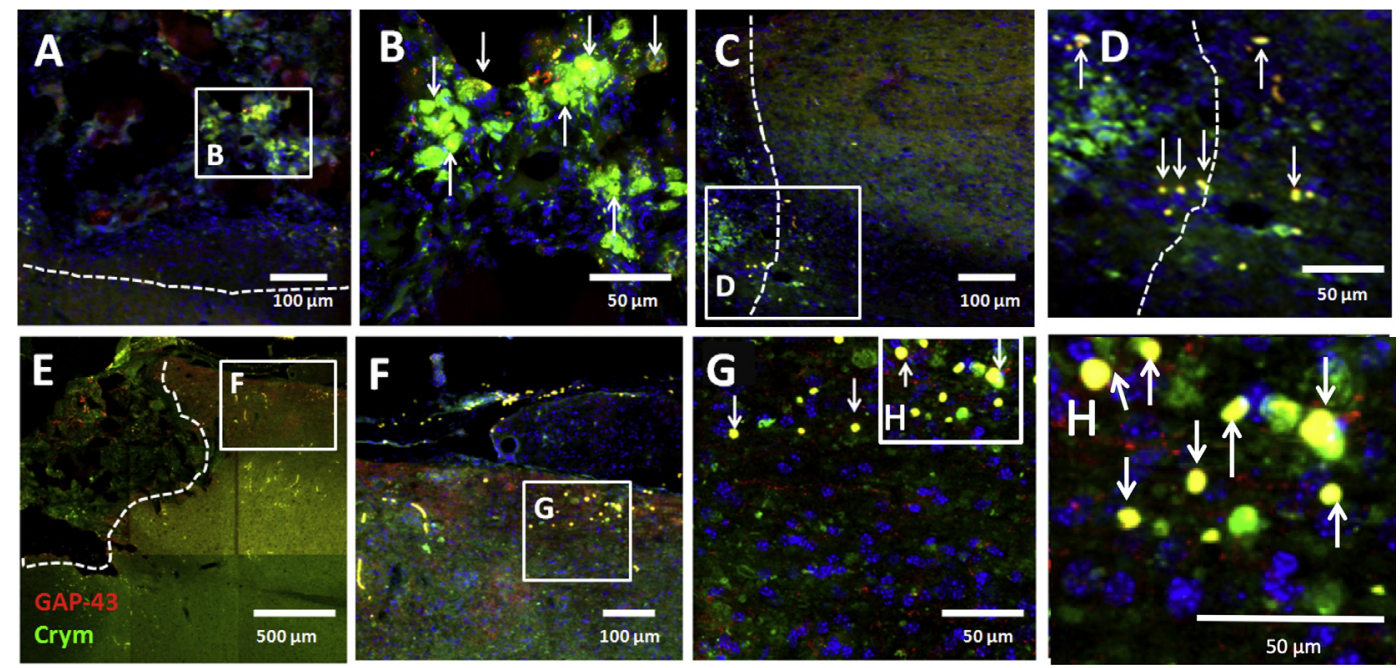

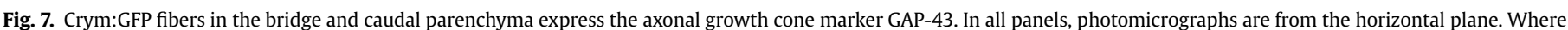

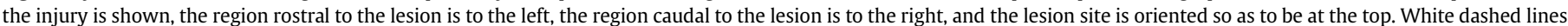

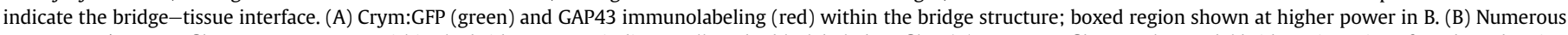

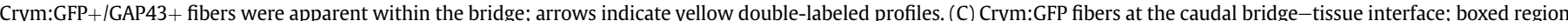

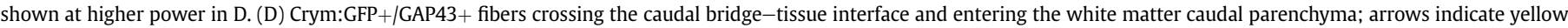

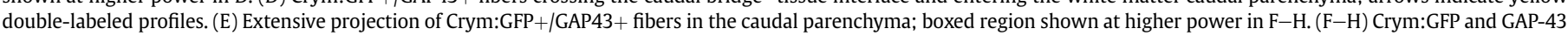

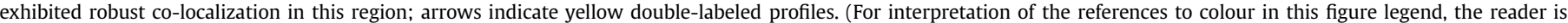
referred to the web version of this article.)
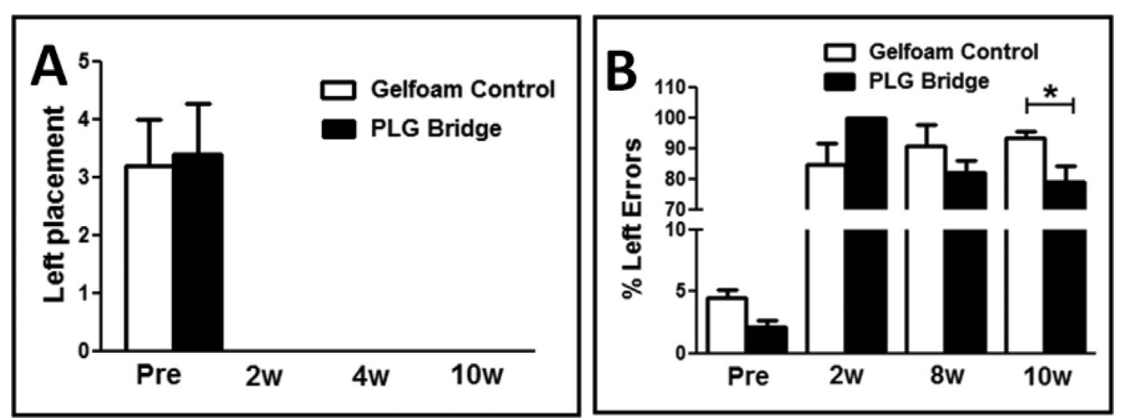

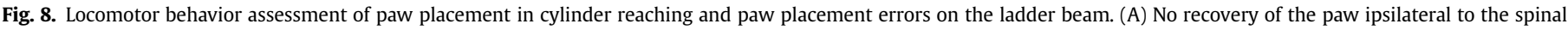

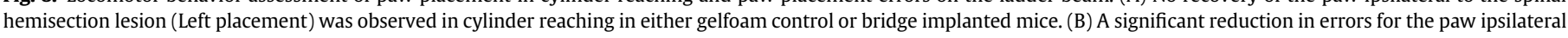

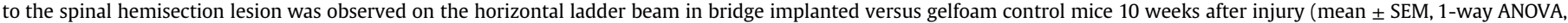
$\mathrm{F}(7,29) \mathrm{p}<0.0001$; *one-tail t-test $\mathrm{p}=0.02$ ).

visualization of Crym:GFP transgene expression in the corticospinal tract, it is likely that the majority of GFP observed in animals with implanted bridges originated in this tract, however, we do not exclude the potential contribution of other descending tracts to spinal cord GFP reporter labeling.

Animals receiving PLG bridges exhibited an increase in NF-200 rostral to the bridge, suggesting that bridge implantation reduced axonal dieback or enhanced growth in this region. Animals receiving PLG bridges also exhibited an increase in NF-200 rostral, within, and caudal to the bridge, which was frequently co-localized with Crym:GFP reporter expression, suggesting that bridge implantation was permissive for axonal regeneration. While biomaterial approaches do not uniformly result in axon sparing, there is precedent for such an observation $(9,34)$; accordingly, it is possible that PLG bridge implantation contributed to sparing of axons and or parenchyma adjacent to the implantation site in addition to enabling axonal growth. The axonal cytoskeleton is comprised of three major neurofilament subunits, the light chain (NF-L/NF-70, 68-70 kDa in size), medium chain (NF-M/NF-160, 145-160 kDa in size), and heavy chain (NF-H/NF-200, 200-220 kDa in size). Axonal maturation accompanied by transition from selective early expression of NF-L and NF-M to the appearance of NF-H, which is thought to play a key role in both axon stabilization and continued extension [56,57]. Future studies investigating other neurofilament subunits at different times post-SCI and bridge implantation may therefore provide insight in comparing developmental tract formation with adult axon extension. Importantly, however, our data did not identify a non-specific non-axonal origin for Crym:GFP labeling. In particular, although a small fraction of NF-200 negative GFP reporter staining was accounted for by co-localization with the macrophage marker F4/80, the majority of GFP labeling was not associated with debris phagocytosis. Further, many GFP positive axons rostral to, within, and caudal to the bridge were positive for GAP-43, which is localized to the plasma membrane of axons and growth cones and involved in axonal regeneration and elongation as an axonal transport membrane protein [58-60]. Taken together, these data demonstrate that GFP expression was highly localized to descending regenerating axons not only entering the bridge rostrally, but also exiting the bridge caudally, a novel observation for a biodegradable implantable bridge in SCI. 
A principal reason for selection of a cervical injury site in this study was because we hypothesized that the demonstrated potential for reorganization of the CST in this region could increase the likelihood of observation of a regenerative response after bridge implantation [61]. Surprisingly, we found Crym:GFP/GAP-43 positive axon segments at distances greater than $2500 \mu \mathrm{m}$ beyond the caudal end of the PLG bridge in the adult spinal cord. This observation suggests that, by 10 weeks post-injury, descending fibers were capable of extending greater than $3500 \mu \mathrm{m}$ past the location at which they were severed. Previous work has shown that manipulation of either the growth state of projection neurons, e.g. via deletion of PTEN or cortical PKC inhibition [62-64], or the inhibitory microenvironment of the spinal cord, e.g. via administration of chrondroitenase or myelin proteins or signaling pathways [9,65-68], can promote regeneration in the adult CNS after SCI. However, to our knowledge, long distance regeneration has not been previously observed in a model where neither the projection neurons nor the spinal microenvironment were directly manipulated.

A second reason for the selection of a cervical injury site was to establish a model for bridge implantation in which sensitive quantitative tasks to assess functional recovery of the ipsilateral limb could be employed. In this regard, the discrete segmental organization of the cervical spinal cord motor neuron columns yields improved sensitivity for assessment of recovery in functional behavioral measures in comparison with thoracic SCI. Additionally, forelimb assessment in cervical SCI models parallels key features of locomotor movement in the human clinical setting [69-71]. Finally, the contribution of descending tracts to forelimb task function is relatively well-defined, and includes both the corticospinal (CST) and rubrospinal (RST) tracts [72], and a direct correlation between CST damage as assessed in diffusion tensor MRI imaging and loss of manual dexterity has been reported in human SCI [3].

Sustained deficits in stepping and cylinder tasks have previously been described in rats receiving a C3-4 lateral hemisection [70]; neuroanatomical tracing of the ipsilateral CST in this study demonstrated a predictive relationship between forelimb stepalternation and lesion severity. Accordingly, we selected a horizontal ladder task, in which the stepping ability is assessed by the number of errors made during rung crossing [44,73], and a cylinder paw preference task, in which reaching ability is assessed by number of paw placements $[74,75]$. Animals receiving PLG bridges exhibited a reduction in errors on the horizontal ladder task, indicative of recovery of function. However, no change in paw placements was observed on the cylinder task. Motor neuron columns controlling the muscles of the shoulder extend through $\mathrm{C} 2-\mathrm{C} 5$, those of the upper arm extend through $\mathrm{C} 4$ to $\mathrm{C} 8$, and those of the lower arm extend through C5-T1 [76]. Cylinder reaching is dependent on a combination of shoulder as well as upper and lower arm function; the absence of recovery on this task is consistent with significant disruption of the C4-5 shoulder and upper arm motor neuron pool by the $1.25 \mathrm{~mm}$ lesion, and with previous observations in our laboratory using a unilateral C5 contusion model. Horizontal ladder beam errors in contrast, may be more dependent on arm and paw function, which could reflect axons that have reached C6. These data could therefore reflect reinnervation of ipsilateral targets by fibers exiting the bridge and entering the spared caudal parenchyma. Critically, however, the potential contribution of minimization of tissue loss and glial scar formation cannot be excluded, and further studies investigating the mechanisms of recovery, including the establishment of direct evidence of synaptic/functional connectivity, are necessary. However, a principal priority for individuals with cervical level SCI is to regain partial or full function of the arm and/or hand, which can significantly improve independence and quality of life $[70,77]$.
Accordingly, the potential of an implanted bridge alone, that is, without combination with trophic factors or seeded cell populations, to promote both regeneration and recovery of function is both novel and clinically relevant for $\mathrm{SCI}$.

\section{Conclusion}

Overall, these data suggest a surprising capacity for regenerative growth of the CST in a biomaterial bridge, even in the absence of targeted manipulation of either intrinsic growth programs in cortical projection neurons or extrinsic growth inhibitory signals in the post-SCI microenvironment. Further analysis of the cellular microenvironment within the bridge and at the bridge-tissue interface may yield additional insight into the permissive state provided for axonal growth in this paradigm, as well as additional targets for improving axonal growth and/or re-entry into the spinal cord below the lesion.

\section{Acknowledgments}

We thank Rebecca Nishi and Hong-Li Liu for assistance with animal surgeries, and Jovanny Lucero for assistance with behavioral assessments. Funding for this work was provided by $\mathrm{NIH}$ 2R01EB005678-05 (LDS, AJA and BJC) and the Christopher and Dana Reeve Foundation (CDRF) (AJA).

\section{Appendix A. Supplementary data}

Supplementary data related to this article can be found at http:// dx.doi.org/10.1016/j.biomaterials.2015.05.032.

\section{References}

[1] J. Nielson, M. Strong, O. Steward, A reassessment of whether cortical motor neurons die following spinal cord injury, J. Comp. Neurol. 519 (2011) 2852-2869.

[2] M. Tuszynski, O. Steward, Concepts and methods for the study of axonal regeneration in the CNS, Neuron 74 (2012) 777-791.

[3] P. Freund, T. Schneider, Z. Nagy, C. Hutton, N. Weiskopf, K. Friston, et al., Degeneration of the injured cervical cord is associated with remote changes in corticospinal tract integrity and upper limb impairment, PloS One 7 (2012).

[4] F. Bareyre, M. Kerschensteiner, O. Raineteau, T. Mettenleiter, O. Weinmann, M. Schwab, The injured spinal cord spontaneously forms a new intraspinal circuit in adult rats, Nat. Neurosci. 7 (2004) 269-277.

[5] K. Fouad, V. Pedersen, M. Schwab, C. Brösamle, Cervical sprouting of corticospinal fibers after thoracic spinal cord injury accompanies shifts in evoked motor responses, Curr. Biol.: CB 11 (2001) 1766-1770.

[6] E. Rosenzweig, G. Courtine, D. Jindrich, J. Brock, A. Ferguson, S. Strand, et al., Extensive spontaneous plasticity of corticospinal projections after primate spinal cord injury, Nat. Neurosci. 13 (2010) 1505-1510.

[7] L. Schnell, A. Hunanyan, W. Bowers, P. Horner, H. Federoff, M. Gullo, et al, Combined delivery of Nogo-A antibody, neurotrophin-3 and the NMDA-NR2d subunit establishes a functional 'detour' in the hemisected spinal cord, Eur. J. Neurosci. 34 (2011) 1256-1267.

[8] E. Joosten, Biodegradable biomatrices and bridging the injured spinal cord: the corticospinal tract as a proof of principle, Cell Tissue Res. 349 (2012) 375-395.

[9] J. Fawcett, M. Schwab, L. Montani, N. Brazda, H. Müller, Defeating inhibition of regeneration by scar and myelin components, Handb. Clin. Neurol. 109 (2012) $503-522$.

[10] S. Neumann, C. Woolf, Regeneration of dorsal column fibers into and beyond the lesion site following adult spinal cord injury, Neuron 23 (1999) 83-91.

[11] W. Plunet, B. Kwon, W. Tetzlaff, Promoting axonal regeneration in the central nervous system by enhancing the cell body response to axotomy, J. Neurosci. Res. 68 (2002) 1-6.

[12] J. Silver, J. Miller, Regeneration beyond the glial scar, Nat. Rev. Neurosci. 5 (2004) 146-156.

[13] M. Bunge, P. Wood, Realizing the maximum potential of Schwann cells to promote recovery from spinal cord injury, Handb. Clin. Neurol. 109 (2012) 523-540.

[14] S. David, A. Aguayo, Axonal elongation into peripheral nervous system "bridges" after central nervous system injury in adult rats, Science (New York, NY) 214 (1981) 931-933.

[15] R. Mueller, I. Ferreira, K. Pawar, N. Weidner, Application of hydrogels to rewire the injured spinal cord, in: S. David (Ed.), Handbook of Hydrogels: Properies, 
Preparation and Application, Nova Science Publisher, 2009, pp. 397-425.

[16] E. Ansorena, P. De Berdt, B. Ucakar, T. Simón-Yarza, D. Jacobs, O. Schakman, et al., Injectable alginate hydrogel loaded with GDNF promotes functional recovery in a hemisection model of spinal cord injury, Int. J. Pharm. 455 (2013) $148-158$.

[17] D. Cigognini, A. Satta, B. Colleoni, D. Silva, M. Donegà, S. Antonini, et al, Evaluation of early and late effects into the acute spinal cord injury of an injectable functionalized self-assembling scaffold, PloS One 6 (2011).

[18] V. King, A. Alovskaya, D. Wei, R. Brown, J. Priestley, The use of injectable forms of fibrin and fibronectin to support axonal ingrowth after spinal cord injury, Biomaterials 31 (2010) 4447-4456.

[19] P. Lu, Y. Wang, L. Graham, K. McHale, M. Gao, D. Wu, et al., Long-distance growth and connectivity of neural stem cells after severe spinal cord injury, Cell 150 (2012) 1264-1273.

[20] K. Sharp, A. Dickson, S. Marchenko, K. Yee, P. Emery, I. Laidmåe, et al., Salmon fibrin treatment of spinal cord injury promotes functional recovery and density of serotonergic innervation, Exp. Neurol. 235 (2012) 345-356.

[21] V. Tysseling-Mattiace, V. Sahni, K. Niece, D. Birch, C. Czeisler, M. Fehlings, et al. Self-assembling nanofibers inhibit glial scar formation and promote axon elongation after spinal cord injury, J. Neurosci.: Off. J. Soc. Neurosci. 28 (2008) 3814-3823.

[22] M. Daud, K. Pawar, F. Claeyssens, A. Ryan, J. Haycock, An aligned 3D neuronalglial co-culture model for peripheral nerve studies, Biomaterials 33 (2012) 5901-5913.

[23] K. Kang, D.Y. Kim, S. Yoon, J. Lee, B. Lee, J. Kwon, et al., Tissue engineered regeneration of completely transected spinal cord using human mesenchyma stem cells, Biomaterials 33 (2012) 4828-4835.

[24] L. Novikova, J. Pettersson, M. Brohlin, M. Wiberg, L. Novikov, Biodegradable poly-beta-hydroxybutyrate scaffold seeded with Schwann cells to promote spinal cord repair, Biomaterials 29 (2008) 1198-1206.

[25] H. Olson, G. Rooney, L. Gross, J. Nesbitt, K. Galvin, A. Knight, et al., Neural stem cell- and Schwann cell-loaded biodegradable polymer scaffolds suppor axonal regeneration in the transected spinal cord, Tissue Eng. Part A 15 (2009) 1797-1805.

[26] K. Pawar, R. Mueller, M. Caioni, P. Prang, U. Bogdahn, W. Kunz, et al. Increasing capillary diameter and the incorporation of gelatin enhance axon outgrowth in alginate-based anisotropic hydrogels, Acta Biomater. 7 (2011) 2826-2834.

[27] P. Prang, R. Muller, A. Eljaouhari, K. Heckmann, W. Kunz, T. Weber, et al., The promotion of oriented axonal regrowth in the injured spinal cord by alginatebased anisotropic capillary hydrogels, Biomaterials 27 (2006) 3560-3569.

[28] A. Thomas, M. Kubilius, S. Holland, S. Seidlits, R. Boehler, A. Anderson, et al., Channel density and porosity of degradable bridging scaffolds on axon growth after spinal injury, Biomaterials 34 (2013) 2213-2220.

[29] H. Tuinstra, M. Aviles, S. Shin, S. Holland, M. Zelivyanskaya, A. Fast, et al., Multifunctional, multichannel bridges that deliver neurotrophin encoding lentivirus for regeneration following spinal cord injury, Biomaterials 33 (2012) 1618-1626.

[30] L. De Laporte, Y. Yang, M. Zelivyanskaya, B. Cummings, A. Anderson, L. Shea, Plasmid releasing multiple channel bridges for transgene expression after spinal cord injury, Mol. Ther.: J. Am. Soc. Gene Ther. 17 (2009) 318-326.

[31] Y. Yang, L. De Laporte, M. Zelivyanskaya, K. Whittlesey, A. Anderson, B. Cummings, et al., Multiple channel bridges for spinal cord injury: cellula characterization of host response, Tissue Eng. Part A 15 (2009) 3283-3295.

[32] Y. Teng, E. Lavik, X. Ou, K. Park, J. Ourednik, D. Zurakowski, et al., Functiona recovery following traumatic spinal cord injury mediated by a unique polymer scaffold seeded with neural stem cells, Proc. Natl. Acad. Sci. U. S. A. 99 (2002) 3024-3029.

[33] Y. Suzuki, M. Kitaura, S. Wu, K. Kataoka, K. Suzuki, K. Endo, et al., Electrophysiological and horseradish peroxidase-tracing studies of nerve regeneration through alginate-filled gap in adult rat spinal cord, Neurosci. Lett. 318 (2002) 121-124.

[34] C. Brösamle, M. Schwab, Cells of origin, course, and termination patterns of the ventral, uncrossed component of the mature rat corticospinal tract, J. Comp. Neurol. 386 (1997) 293-303.

[35] E. Joosten, A. Gribnau, P. Dederen, An anterograde tracer study of the developing corticospinal tract in the rat: three components, Brain Res. 433 (1987) $121-130$.

[36] O. Steward, B. Zheng, C. Ho, K. Anderson, M. Tessier-Lavigne, The dorsolatera corticospinal tract in mice: an alternative route for corticospinal input to caudal segments following dorsal column lesions, J. Comp. Neurol. 472 (2004) $463-477$.

[37] F. Bareyre, M. Kerschensteiner, T. Misgeld, J. Sanes, Transgenic labeling of the corticospinal tract for monitoring axonal responses to spinal cord injury, Nat. Med. 11 (2005) 1355-1360.

[38] J. Gorski, T. Talley, M. Qiu, L. Puelles, J. Rubenstein, K. Jones, Cortical excitatory neurons and glia, but not GABAergic neurons, are produced in the Emx1expressing lineage, J. Neurosci.: Off. J. Soc. Neurosci. 22 (2002) 6309-6314.

[39] N. Aoki, K. Ito, M. Ito, mu-Crystallin, thyroid hormone-binding protein, is expressed abundantly in the murine inner root sheath cells, J. Invest. Dermatol. 115 (2000) 402-405.

[40] S. Gong, C. Zheng, M.L. Doughty, K. Losos, N. Didkovsky, U.B. Schambra, et al., A gene expression atlas of the central nervous system based on bacterial artificial chromosomes, Nature 425 (2003) 917-925.

[41] E.F. Schmidt, L. Kus, S. Gong, N. Heintz, BAC transgenic mice and the GENSAT database of engineered mouse strains, Cold Spring Harb. Protoc. 2013 (2013).

[42] M. Moore, J. Friedman, E. Lewellyn, S. Mantila, A. Krych, S. Ameenuddin, et al. Multiple-channel scaffolds to promote spinal cord axon regeneration, Biomaterials 27 (2006) 419-429.

[43] Y. Yang, L. De Laporte, C. Rives, J.-H. Jang, W.-C. Lin, K. Shull, et al., Neurotrophin releasing single and multiple lumen nerve conduits, J. Control. Release: Off. J. Control. Release Soc. 104 (2005) 433-446.

[44] B. Cummings, C. Engesser-Cesar, G. Cadena, A. Anderson, Adaptation of a ladder beam walking task to assess locomotor recovery in mice following spinal cord injury, Behav. Brain Res. 177 (2007) 232-241.

[45] O. Steward, B. Zheng, M. Tessier-Lavigne, False resurrections: distinguishing regenerated from spared axons in the injured central nervous system, J. Comp. Neurol. 459 (2003) 1-8.

[46] E. Collyer, A. Catenaccio, D. Lemaitre, P. Diaz, V. Valenzuela, F. Bronfman, et al., Sprouting of axonal collaterals after spinal cord injury is prevented by delayed axonal degeneration, Exp. Neurol. 261 (2014) 451-461.

[47] H.M. Tuinstra, D.J. Margul, A.G. Goodman, R.M. Boehler, S.J. Holland, M.L. Zelivyanskaya, et al., Long-term characterization of axon regeneration and matrix changes using multiple channel bridges for spinal cord regeneration, Tissue Eng. Part A 20 (2014) 1027-1037.

[48] Foundation CaDR, One Degree of Separation: Paralysis and Spinal Cord Injury in the United States, 2009.

[49] B.A. Kakulas, Neuropathology: the foundation for new treatments in spinal cord injury, Spinal Cord. 42 (2004) 549-563.

[50] Center NSCIS, Spinal Cord Injury Facts and Figures at a Glance, 2012.

[51] J.A. Blair, D.R. Possley, J.L. Petfield, A.J. Schoenfeld, R.A. Lehman, J.R. Hsu, et al., Military penetrating spine injuries compared with blunt, Spine J. Off. J. North Am. Spine Soc. 12 (2012) 762-768.

[52] J.A. Willen, U.H. Gaekwad, B.A. Kakulas, Burst fractures in the thoracic and lumbar spine. A clinico-neuropathologic analysis, Spine 14 (1989) $1316-1323$.

[53] J.W. Fawcett, A. Curt, J.D. Steeves, W.P. Coleman, M.H. Tuszynski, D. Lammertse, et al., Guidelines for the conduct of clinical trials for spinal cord injury as developed by the ICCP panel: spontaneous recovery after spinal cord injury and statistical power needed for therapeutic clinical trials, Spinal Cord. 45 (2007) 190-205.

[54] P. Rhee, E.J. Kuncir, L. Johnson, C. Brown, G. Velmahos, M. Martin, et al. Cervical spine injury is highly dependent on the mechanism of injury following blunt and penetrating assault, J. Trauma 61 (2006) 1166-1170.

[55] C. Lang, X. Guo, M. Kerschensteiner, F. Bareyre, Single collateral reconstructions reveal distinct phases of corticospinal remodeling after spinal cord injury, PloS One 7 (2012).

[56] Q. Liu, F. Xie, S. Siedlak, A. Nunomura, K. Honda, P. Moreira, et al., Neurofilament proteins in neurodegenerative diseases, Cell. Mol. Life Sci.: CMLS 61 (2004) 3057-3075.

[57] J. Yabe, F. Wang, T. Chylinski, T. Katchmar, T. Shea, Selective accumulation of the high molecular weight neurofilament subunit within the distal region of growing axonal neurites, Cell Motil. Cytoskelet. 50 (2001) 1-12.

[58] H. Baba, Y. Maezawa, S. Imura, N. Kawahara, K. Nakahashi, K. Tomita, Quantitative analysis of the spinal cord motoneuron under chronic compression: an experimental observation in the mouse, J. Neurol. 243 (1996) 109-116.

[59] L. Benowitz, A. Routtenberg, GAP-43: an intrinsic determinant of neuronal development and plasticity, Trends Neurosci. 20 (1997) 84-91.

[60] J. Skene, M. Willard, Axonally transported proteins associated with axon growth in rabbit central and peripheral nervous systems, J. Cell Biol. 89 (1981) 96-103.

[61] M. Oudega, M. Perez, Corticospinal reorganization after spinal cord injury, J. Physiol. 590 (2012) 3647-3663.

[62] K. Liu, Y. Lu, J. Lee, R. Samara, R. Willenberg, I. Sears-Kraxberger, et al., PTEN deletion enhances the regenerative ability of adult corticospinal neurons, Nat. Neurosci. 13 (2010) 1075-1081.

[63] D.L. Moore, M.G. Blackmore, Y. Hu, K.H. Kaestner, J.L. Bixby, V.P. Lemmon, et al., KLF family members regulate intrinsic axon regeneration ability, Science 326 (2009) 298-301.

[64] X. Wang, J. Hu, Y. She, G. Smith, X.-M. Xu, Cortical PKC Inhibition Promotes Axonal Regeneration of the Corticospinal Tract and Forelimb Functional Recovery after Cervical Dorsal Spinal Hemisection in Adult Rats, Cerebral Cortex, New York, NY, 1991, p. 2013.

[65] F. Akbik, W. Cafferty, S. Strittmatter, Myelin associated inhibitors: a link between injury-induced and experience-dependent plasticity, Exp. Neurol. 235 (2012) 43-52.

[66] L. McKerracher, P. Guertin, Rho as a target to promote repair: translation to clinical studies with cethrin, Curr. Pharm. Des. 19 (2013) 4400-4410.

[67] L. Moon, R. Asher, K. Rhodes, J. Fawcett, Regeneration of CNS axons back to their target following treatment of adult rat brain with chondroitinase $A B C$, Nat. Neurosci. 4 (2001) 465-466.

[68] R.-R. Zhao, J. Fawcett, Combination treatment with chondroitinase ABC in spinal cord injury-breaking the barrier, Neurosci. Bull. 29 (2013) 477-483.

[69] W. Alilain, K. Horn, H. Hu, T. Dick, J. Silver, Functional regeneration of respiratory pathways after spinal cord injury, Nature 475 (2011) 196-200.

[70] Z. Khaing, S. Geissler, S. Jiang, B. Milman, S. Aguilar, C. Schmidt, et al., Assessing forelimb function after unilateral cervical spinal cord injury: novel forelimb tasks predict lesion severity and recovery, J. Neurotrauma 29 (2012) 488-498.

[71] M. Starkey, K. Bartus, A. Barritt, E. Bradbury, Chondroitinase ABC promotes 
compensatory sprouting of the intact corticospinal tract and recovery of forelimb function following unilateral pyramidotomy in adult mice, Eur. J. Neurosci. 36 (2012) 3665-3678.

[72] C. Hurd, N. Weishaupt, K. Fouad, Anatomical correlates of recovery in single pellet reaching in spinal cord injured rats, Exp. Neurol. 247 (2013) 605-614.

[73] Y. Manso, M. Serra, G. Comes, M. Giralt, J. Carrasco, N. Cols, et al., The comparison of mouse full metallothionein-1 versus alpha and beta domains and metallothionein-1-to-3 mutation following traumatic brain injury reveals different biological motifs, J. Neurosci. Res. 88 (2010) 1708-1718.

[74] S. Brooks, S. Dunnett, Tests to assess motor phenotype in mice: a user's guide,
Nat. Rev. Neurosci. 10 (2009) 519-529.

[75] R. Iancu, P. Mohapel, P. Brundin, G. Paul, Behavioral characterization of a unilateral 6-OHDA-lesion model of Parkinson's disease in mice, Behav. Brain Res. 162 (2005) 1-10.

[76] J. McKenna, G. Prusky, I. Whishaw, Cervical motoneuron topography reflects the proximodistal organization of muscles and movements of the rat forelimb: a retrograde carbocyanine dye analysis, J. Comp. Neurol. 419 (2000) 286-296.

[77] K. Anderson, Targeting recovery: priorities of the spinal cord-injured population, J. Neurotrauma 21 (2004) 1371-1383. 